\title{
THE INTERRELATIONS OF THE SERUM PROTEINS IN LIVER DAMAGE, WITH SPECIAL REFERENCE TO THE THYMOL TEST
}

\author{
BY
}

\author{
N. H. MARTIN \\ From St. George's Hospital, London
}

(RECEIVED FOR PUBLICATION, SEPTEMBER 8, 1949)

Maclagan (1944a) demonstrated that phenol and certain derivatives when dissolved in barbiturate buffer at $p \mathrm{H} 7.8$ produced a turbidity with human sera. He observed that under specified conditions sera from normal persons gave a negligible turbidity, and the most marked turbidities occurred with sera from patients suffering from parenchymatous liver damage. The concentrations of phenol or phenol derivative required to produce a given turbidity with any serum diminished as molecular weight increased, but at the same time solubility in an aqueous buffer diminished to such an extent as to limit the range of homologous phenol derivatives that could be used conveniently without the addition of extraneous substances to enhance solubility. As a result of his investigations Maclagan chose thymol as the most suitable derivative, and defined the conditions for carrying out the test and the degree of turbidity which might be regarded as outside normal limits. Maclagan (1944c) never claimed that his test was specific. It is none the less striking that sera in which the highest thymol readings are recorded are predominantly those from patients suffering from liver damage.

In 1947 Maclagan and Bunn employed electrophoresis to investigate the contributions of the individual components of the serum protein mosaiz to the thymol turbidity test. They observed that turbidity occurred when $\gamma$-globulin from hepatitis serum was added to the thymol reagent and that human albumin from normal persons prevented this reaction. Albumin separated by electrophoresis from the serum of patients suffering from infective hepatitis did not prevent the reaction. They observed, further, that $\gamma$-globulin separated from the serum of normal persons would produce turbidity with the thymol reagent, but that the minimal concentration required was greater than $\gamma$-globulin from the serum of patients suffering from hepatitis. Neither normal $\alpha$ - and $\beta$-globulin, nor $\alpha$ - and $\beta$-globulin separated from the serum of patients suffering from hepatitis, gave turbidities with the thymol reagent, either before or after sensitization of the reagent with cephalin ; $\alpha$ - and $\beta$-globulin tended to sensitize the reaction of the thymol reagent with $\gamma$-globulin.

Kunkel and Hoagland (1947), studying the reaction of normal and pathological sera, demonstrated that when a serum giving a markedly positive reaction is extracted with ether the thymol reaction is markedly reduced, the example quoted in their paper showing a reduction from 22 to 9 units. They examined sera by electrophoresis before and after flocculation with the thymol reagent, and showed that such changes as there were affected the $\beta$-fraction principally. Finally, in six sera they examined, in which the thymol reaction was above 25 units, the lipid content was $100 \%$ higher than in six sera with a thymol reaction below 15 units. Their findings stress the significance of the lipid content of the serum and imply that the $\beta$-globulin is the active participant in the test.

Cohen and Thompson (1947) produced further data which they interpreted as indicating that $\beta$ globulin was the active agent in the thymol test.

The experiments of Wunderly and Wuhrmann (1947) in which $\gamma$-globulin was added to normal serum, thereby producing turbidity with the thymol reagent, seem to support Maclagan's conclusion that $\gamma$-globulin of itself can be responsible for the production of turbidity with the thymol reagent. Maclagan (1944b) pointed out that lipids might increase the sensitivity of the reaction and, indeed, he demonstrated that the precipitate from 
the reaction contains cholesterol, phospholipid, and protein.

The fullest understanding of the alterations in the circulating proteins which result in positive thymol turbidity tests may throw light on the biochemical lesions underlying some forms of failure of liver function.

\section{Material}

In the past years I have been accumulating observations on the circulating proteins in the sera of patients suffering from liver failure. From this material the selected data seemed particularly suitable for the study of the relationship of the thymol turbidity reaction to the nature of the proteins circulating in conditions primarily involving the liver.

The material may be divided into two groups: one, from patients suffering from, and recovering from, acute attacks of infective hepatitis; the other, from patients suffering from chronic progressive liver failure.

The $\beta$ - and $\gamma$-globulins which formed the bases of special study were separated by electrophoresis from the sera of two patients suffering from acute hepatitis in whom the thymol turbidity values ranged at 32 and 36 units respectively.

\section{Methods}

The thymol turbidity tests were carried out according to the technique laid down by Maclagan (1944b).

The electrophoretic analyses were carried out in the apparatus designed by Tiselius (1937), us ng the $11-\mathrm{cm}$. analytical cell, and in a barbiturate buffer of 0.1 ionic strength and $p \mathrm{H} 8.6$ and in a phosphate buffer at 0.2 ionic strength and $p \mathbf{H ~ 8 . 0 . ~ A l l ~}$ protein concentrations were adjusted to $2.0 \%$ and dialysed against successive changes of buffer before analysis.

Ultracentrifugal analyses were carried out in the oil-driven centrifuge designed by Svedberg.

Nitrogen was estimated by the standard microKjeldahl technique and cholesterol by a modification of the method of Schoenheimer and Sperry (1934).

\section{Results}

Acute Infective Hepatitis.-The proportion of $\gamma$-globulin in the group of patients with acute infective hepatitis is shown plotted against thymol units in Fig. 1.

The ratio of albumin to $\gamma$-globulin and of albumin to $\beta+\gamma$-globulin from the same group is shown plotted in Fig. 2. There appears to be poor
ACUTE PARENCHYMATOUS DAMAGE

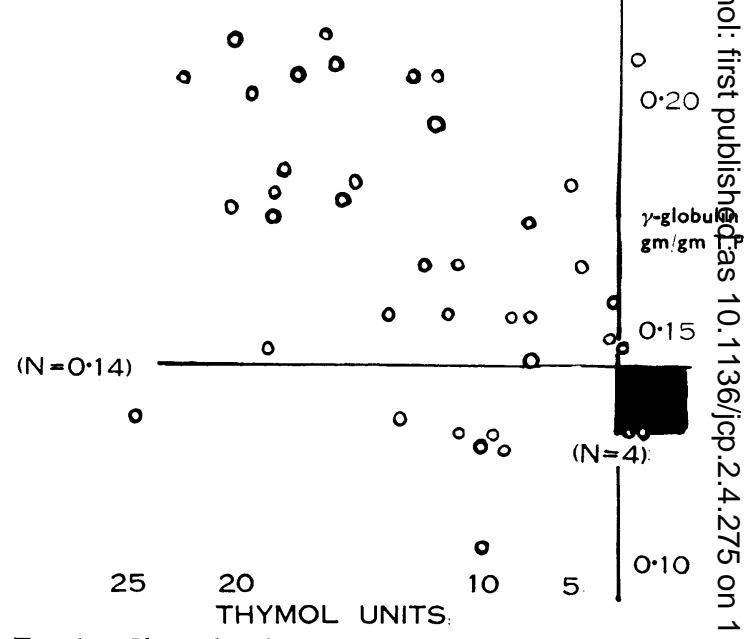

FIG. 1.-Chart showing $\gamma$-globulin (as percentage of total $Z$ protein) plotted against thymol turbidity in acute? infective hepatitis.

correlation between the $\gamma$-globulin concentration or the $\beta+\gamma$-globulin concentration and thymol $\overrightarrow{0}$ units, but there is a definite correlation betwegn the ratio of albumin to globulin and thymol uni

From two sera the $\beta+\gamma$-globulin fractions we separated and pooled. They were then divide

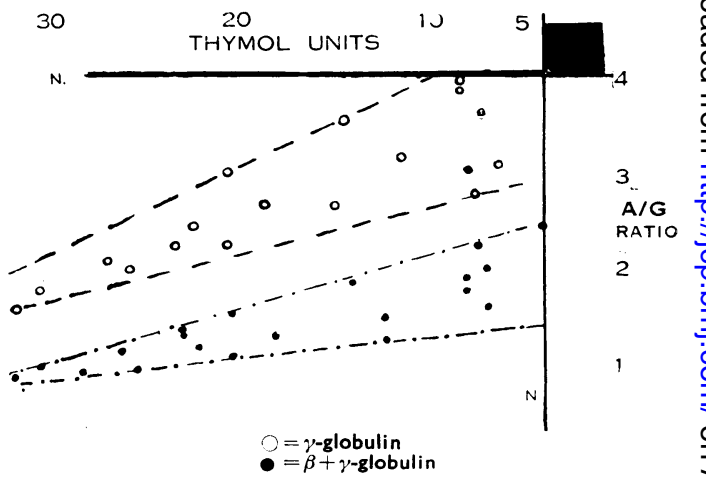

FIG. 2.-Albumin $/ \gamma$-globulin and albumin $/ \beta+\gamma$-globu- $\frac{D}{0}$ lin ratios plotted against thymol turbidity in acute infective hepatitis.

into aliquot portions and diluted with an equal 0 volume of saline in which had been dissolved vary- $\omega$ ing concentrations of crystalline human albumin

The results of the thymol turbidity test on theo solutions so obtained are shown in Fig. 3. The thymol turbidity in units is plotted against the? ratio of albumin nitrogen to $\beta+\gamma$-globulin $\square$ nitrogen. It will be seen that the ability of the $\frac{0}{+}$ globulin to form a turbid solution with the thymol 


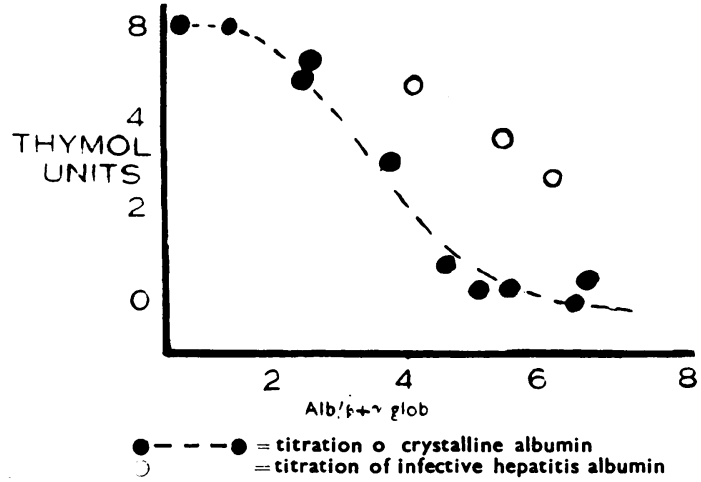

FIG. 3.-Effect of albumin in inhibiting positive thymol effect of $\beta+\gamma$-globulin in infective hepatitis with (a) normal crystalline albumin and (b) albumin from infective hepatitis.

reagent could be inhibited by pure albumin and that the extent of inhibition increased in proportion to the ratio of albumin to globulin. As has been previously recorded this reaction is not species specific (Martin, 1948), parallel effects being obtained with bovine and horse albumin. Three points are also shown of experiments in which albumin, separated from a case of infective hepatitis, was substituted for normal albumin.

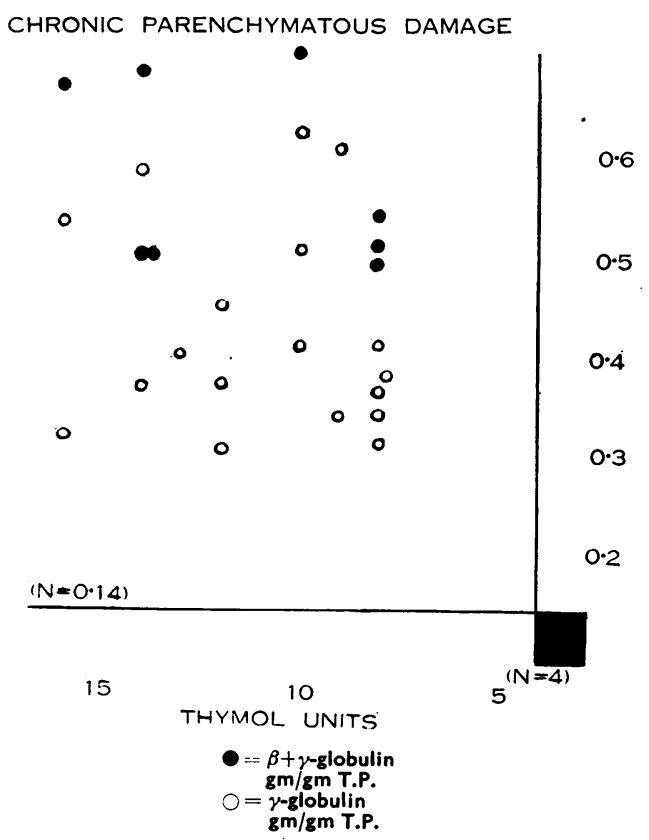

FIG. 4.- $\gamma$-globulin and $\beta+\gamma$-globulin (expressed as percentage of total protein) plotted against thymol turbidity in chronic liver disease.
Chronic Liver Damage. $-\gamma$ - and $\beta+\gamma$-globulin are plotted against thymol units in a group of patients suffering from chronic liver damage in Fig. 4, and the ratio of albumin to $\gamma$-globulin and to $\beta+\gamma$-globulin is shown in Fig. 5 .

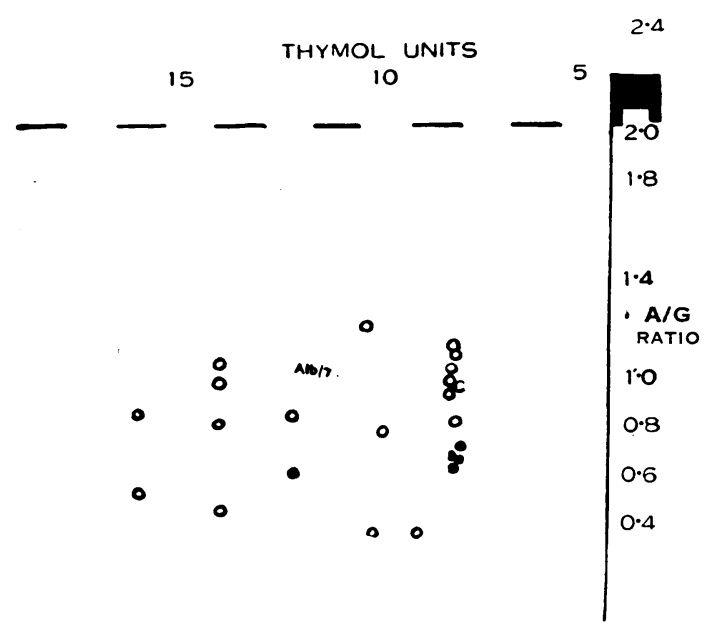

FIG. 5.-Albumin $/ \gamma$-globulin and albumin $/ 3+\gamma$-globulin ratios plotted against thymol turbidity in chronic liver disease.

In contradistinction to the findings, shown in Figs. 1 and 2, neither approach seems to show any significant correlation between protein values and thymol units.

In the course of analysis of the cases of chronic liver damage, two were observed in which spontaneous precipitation of the $\gamma$-globulin took place during electrophoretic analysis.

The precipitation took place in the descending limb only after three hours when there was effective separation of the individual components of the protein mosaic and it is clearly confined to the $\gamma$-globulin. Spontaneous precipitation of this type has never been observed in the serum of patients with acute hepatitis.

Results in Non-hepatic Cases. - " False " positive reactions had been observed occasionally with the thymol reagent during the investigation of cases of hyperglobulinaemia, in which clinical and histological evidence did not suggest any liver damage and other biochemical tests did not support a diagnosis of hepatic insufficiency. These "false" positives were rarely above 10 Maclagan units. Ultracentrifugal analyses from some of these cases have been carried out and demonstrate that in certain instances there are molecules 
present having abnormal sedimentation constants. Fig. 6 shows an analysis of such sera from a case of reticulosis (A), from a case of advanced liver damage (B), and, for comparison, an analysis of serum from a normal person is shown $(\mathrm{N})$.

The presence of molecules with unusually high
They can occur independently of any gross increase or deviation of lipoid in the serum.

The part which lipids play has been stressed by Kunkel and Hoagland (1947). Table $I$ is an analysis of five sera in all of which there was raised cholesterol, inversion of the albumin

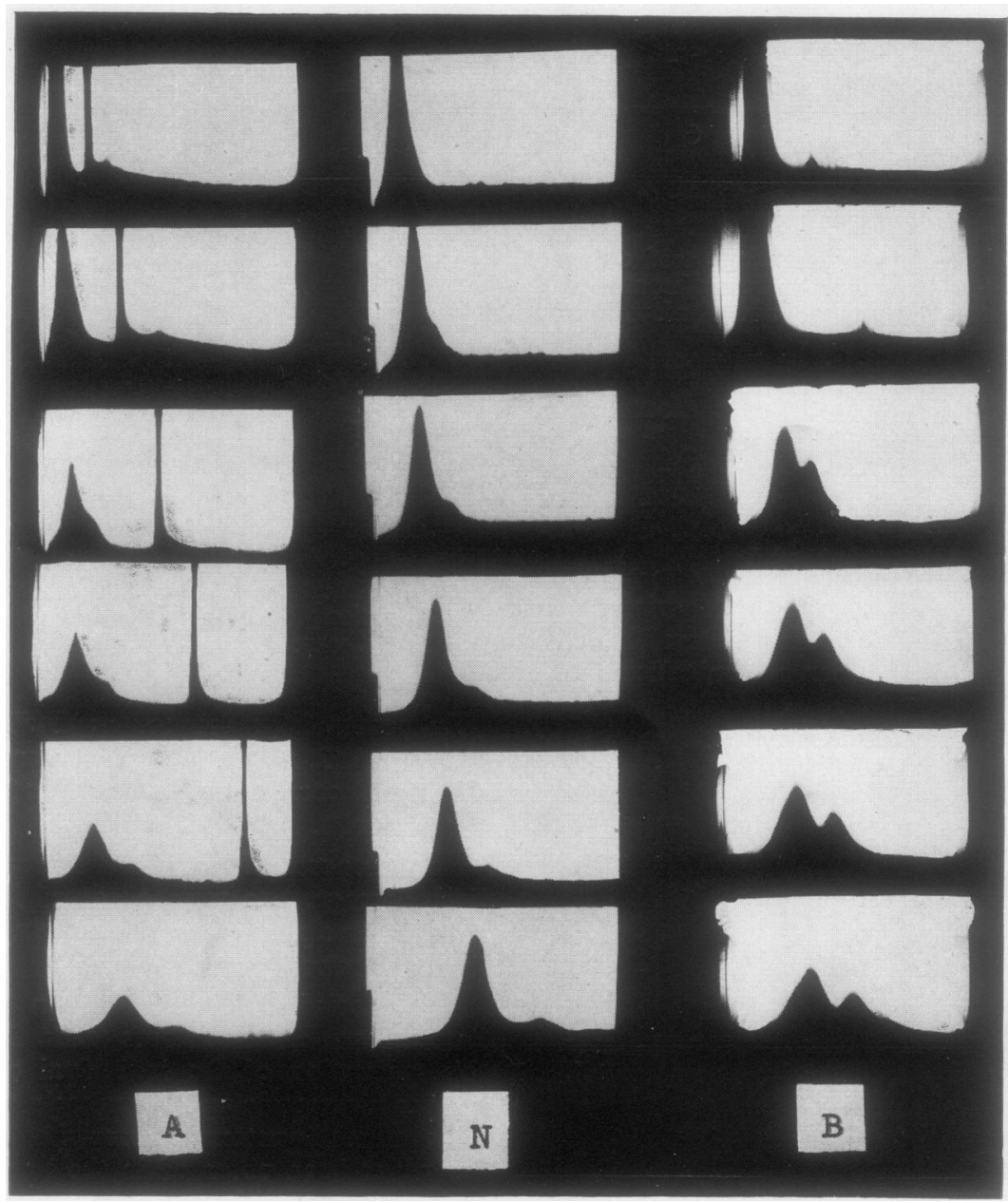

FIG.6.-Ultracentrifugal diagrams showing fractions with high sedimentation constants in serum from a case of reticulosis (A) and advanced liver disease (B). Normal serum $(\mathbf{N})$ is shown for comparison.

sedimentation constants can be seen in both serum $A$ and serum $B$. They are not visible to any appreciable extent in normal sera.

These "macromolecules" were associated with the $\gamma$-globulin fraction in the sera studied. It must be stressed that they are not a constant component of the $\gamma$-fraction in liver disease, nor do they predominate in the $\gamma$-fraction when they do occur. globulin ratio, and a normal reaction to the thymol reagent. Maclagan (1944b) had already shown that the presence of cholesterol increased the sensitivity of the test. It is clear from this table that considerable increases of circulating cholesterol may occur in the presence of inversion of the albumin globulin ratio without producing abnormal turbidities. 
TABLE I

\begin{tabular}{c|c|c|c|c}
\hline Case & $\begin{array}{c}\text { Total Protein } \\
\text { (g./100 ml. } \\
\text { Serum) }\end{array}$ & A/G & $\begin{array}{c}\text { Cholesterol } \\
\text { (mg./100 ml. } \\
\text { Serum) }\end{array}$ & $\begin{array}{c}\text { Thymol } \\
\text { Turbidity } \\
\text { Units }\end{array}$ \\
\hline 1 & 3.5 & 0.3 & 405 & 3.0 \\
2 & 4.2 & 0.33 & 450 & 2.0 \\
3 & 4.4 & 0.25 & 437 & 2.0 \\
4 & 5.2 & 0.34 & 470 & 3.0 \\
5 & 6.2 & 0.5 & 375 & 2.0 \\
Normal & 7.0 & 1.25 & 200 & up to 4.0 \\
\hline
\end{tabular}

\section{Discussion}

The observations presented suggest that, in normal sera, the inability of the thymol reagent to produce more than trace turbidities is due to the interaction of normal albumin with $\gamma$-globulin, for, as Maclagan (1947) has demonstrated, neither $\alpha$ - nor $\beta$-globulins are capable themselves of flocculating with the thymol reagent. The marked turbidities produced in acute infective hepatitis seem to be correlated with the relation of circulating albumin to globulin rather than to the relative increase in the amount of circulating $\gamma$-globulin. Replacement experiments, demonstrating that the addition of normal human albumin to globulins separated from positive sera inhibits turbidity, lend added support to this contention. Similar experiments carried out with albumin separated by electrophoresis from sera of patients suffering from acute hepatitis suggest that in these, at any rate, there are, in addition, qualitative alterations in the circulating albumin which reduce its ability to inhibit the thymol reaction.

It is in acute hepatitis that the highest thymol turbidity readings are commonly encountered, though the disturbance of the albumin globulin ratio is rarely so marked as in chronic liver damage. The evidence suggests that in acute hepatitis qualitative defects in the circulating albumin are important.

The level of thymol turbidities in chronic liver damage bears little relation to the progress of the disease ; nor, in spite of the fact that gross inversions of the albumin globulin ratios are common, is there any correlation with the thymol turbidity readings.
The observation of spontaneous precipitation of the $\gamma$-globulin during the examination of the serum of two cases of advanced chronic liver damage suggested the presence of $\gamma$-globulins inherently unstable in themselves. The demonstration of molecules of abnormal sedimentation rate in the circulating sera of other cases of chronic hepatitis lends added support to the contention that in certain of these patients aberrant globulins contribute to the production of turbidity with the thymol reagent.

These macromolecules are demonstrable occasionally in sera from patients with diseases in which there is no evidence of primary liver damage, the sera frequently giving turbidities above the normal range. The presence of these molecules may be regarded as contributing to a "non-specific reaction." It must be stressed that these macromolecules are rarely present in high concentration and that their presence is by no means constant nor confined to sera from patients with liver damage.

\section{Summary}

Fresh evidence is put forward to support the view that the abnormal thymol turbidities associated with liver damage depend on a disturbance of the intimate relationships of the albumin and globulins in the serum.

Replacement experiments suggest that qualitative defects in albumin are important in the production of the reaction in acute hepatitis.

Abnormal globu ins contribute to some positive reactions which are regarded as " non-specific."

My thanks are due to the officials of the Lister Institute, who have been so generous in granting me research facilities.

A part of this paper was incorporated in a communication to the first International Congress of Biochemistry in August, 1949.

\section{REFERENCES}

Cohen, P. P., and Thompson, F. L. (1947). J. Lab. clin. Med., 32, 475 Kunkel, H. G., and Hoagland, C. L. (1947). J. clin. Invest., 26, 1060 Maclagan, N. F. (1944a). Nature, Lond., 154, 670.

Maclagan, N. F. (1944a). Nature, Lond., (1944c). Brit. med. J., 2, 363.

and Bunn, D. (1947). Biochem. J., 41, 580.

Martin, N. H. (1948). Nature, Lond., 162, 145.

Schoenheimer, R and Sperry, W. M. (1934). J.biol Chem, 106, 745.

Svedberg, Thé., et al. (1940). The Ultracentrifuge. Oxford Üniversity Press. London.
Prond

Tiselius, A. (1937). Biochem. J., 31, 1464.

Wunderly, C., and Wuhrmann, F. (1947). Brit. J. exp. Path., 28, 286. 